\title{
Neuroinflammation in Response to Intracerebral Injections of Different HMGB1 Redox Isoforms
}

\author{
Hannah Aucott ${ }^{a}$ Johan Lundberg ${ }^{b, c}$ Henna Salo ${ }^{a}$ Lena Klevenvall ${ }^{d}$ \\ Peter Damberg $^{\text {b }}$ Lars Ottosson $^{d}$ Ulf Andersson ${ }^{d}$ Staffan Holmin ${ }^{\text {b, } c}$ \\ Helena Erlandsson Harris ${ }^{a}$ \\ ${ }^{a}$ Department of Medicine Solna, Rheumatology Unit, Centre for Molecular Medicine, Karolinska Institutet, \\ Stockholm, Sweden; ${ }^{b}$ Department of Clinical Neuroscience, Karolinska Institutet, Stockholm, Sweden; \\ 'Department of Neuroradiology, Karolinska University Hospital, Stockholm, Sweden; 'Department of Women's \\ and Children's Health, Karolinska Institutet, Stockholm, Sweden
}

\section{Keywords}

HMGB1 · Brain · Redox · Neuroinflammation · Blood-brain barrier

\begin{abstract}
Background: Neuroinflammation triggered by infection or trauma is the cause of central nervous system dysfunction. High-mobility group box 1 protein (HMGB1), released from stressed and dying brain cells, is a potent neuroinflammatory mediator. The proinflammatory functions of HMGB1 are tightly regulated by post-translational redox modifications, and we here investigated detailed neuroinflammatory responses induced by the individual redox isoforms. Methods: Male Dark Agouti rats received a stereotactic injection of saline, lipopolysaccharide, disulfide HMGB1, or fully reduced HMGB1, and were accessed for blood-brain barrier modifications using magnetic resonance imaging (MRI) and inflammatory responses by immunohistochemistry. Results and Conclusions: Significant blood-brain barrier disruption appeared $24 \mathrm{~h}$ after injection of lipopolysaccharide, disulfide HMGB1, or fully reduced HMGB1 compared to controls, as
\end{abstract}

\section{KARGER}

() 2018 S. Karger AG, Basel

E-Mail karger@karger.com

www.karger.com/jin assessed in post-gadolinium T1-weighted MRI images and confirmed by increased uptake of FITC-conjugated dextran. Immunohistochemistry revealed that both HMGB1 isoforms also induced a local production of IL-1 $\beta$. Additionally, disulfide HMGB1 increased major histocompatibility complex class II expression and apoptosis. Together, the results demonstrate that extracellular, cerebral HMGB1 causes significant blood-brain barrier disruption in a redox-independent manner and activates several components of neuroinflammation. Blocking HMGB1 might potentially improve clinical outcome in conditions such as stroke and traumatic brain injury.

(c) 2018 S. Karger AG, Basel

\section{Introduction}

It is increasingly recognised that immune responses in the central nervous system (CNS) contribute significantly to the pathogenesis of both neuroinflammatory and neurodegenerative disorders. Neuroinflammation, involving the activation of glia cells and the production of 
proinflammatory mediators, occurs in response to infection or trauma, including ischemia. An early event in neuro-immune responses is the release of endogenous danger signals or damage-associated molecular pattern molecules (DAMPs) from dying cells. Several molecules belonging to the DAMP family have been identified as possible pathogenic proinflammatory mediators in the CNS, including the high-mobility group box 1 (HMGB1) protein, heat shock proteins, proteins of the peroxiredoxin family, nucleotides, carbohydrates, and lipids $[1,2]$.

HMGB1 is a highly conserved nuclear DNA-binding protein re-discovered as an endogenous inflammatory mediator involved in the pathogenesis of inflammatory and infectious diseases in the late 1990s [3]. Structurally, HMGB1 comprises 215 residues organised into two DNA-binding domains (boxes A and B) and a highly repetitive, negatively charged C-terminal tail. Release of HMGB1, either passively or by active secretion, occurs during cell death or in response to an inflammatory stimulus [3]. Extracellular HMGB1 is reported to interact with several pathogen recognition receptors to promote cell migration and cytokine production. The best characterised of these include toll-like receptor 4 (TLR4) and the receptor for advanced glycation end products (RAGE) [4, 5]. The ability of HMGB1 to interact with multiple unrelated receptors to induce diverse functions is tightly regulated by post-translational redox modification of the three cysteine residues at positions 23,45 , and 106 in the box $\mathrm{A}$ and $\mathrm{B}$ domains $[4,6]$. Three different redox isoforms, with distinct functions, have been detected in vivo: (1) fully reduced HMGB1 (fr-HMGB1), with a thiol group present on each of the cysteine residues, induces cell migration, (2) disulfide HMGB1 (ds-HMGB1), containing a disulfide bridge between $\mathrm{C} 23$ and $\mathrm{C} 45$ and a reduced C106 residue, is a TLR4 ligand, and (3) sulphonyl HMGB1 (ox-HMGB1), where all cysteine residues are terminally oxidised, appears to have lost proinflammatory features and cannot induce cell migration or cytokine production. Additionally, HMGB1 is able to act synergistically with endogenous and exogenous ligands, including CXCL12, IL-1, DNA, and lipopolysaccharide (LPS), to promote inflammation $[7,8]$. The formation of a heterocomplex between fr-HMGB1 and CXCL12 has been detected in vitro using NMR and surface plasmon resonance analysis and in vivo after muscle tissue injury. fr-HMGB1-CXCL12 complexes interact with the chemokine receptor CXCR4 to promote cell migration in a TLR4- and RAGE-independent manner $[6,8]$.

HMGB1 released from neurons and astrocytes induces the production of several inflammatory mediators, in- cluding TNF- $\alpha$, IL-6, and IL-1 $\beta$ [9-11]. The HMGB1RAGE axis has been shown to contribute to brain injuryinduced sickness behaviour in animals [12]. HMGB1 present in the CNS has also been demonstrated to mediate stress-induced neuroinflammatory priming via an NLRP3 inflammasome-dependent mechanism [13]. Weber et al. [13] found that hippocampal ds-HMGB1 released during stress in rats may prime microglia, thereby enhancing proinflammatory responses to subsequent LPS exposure. This effect is inhibited using box A as an antagonist of full-length HMGB1. Peripheral ds- and frHMGB1 also induce hyperalgesia via TLR4 and RAGE, respectively [14], and ds-HMGB1 has been reported to regulate pain in collagen antibody-induced experimental arthritis [15]. In addition, ds-HMGB1 potentiates NMDA-induced $\mathrm{Ca}^{2+}$ influx via TLR4 in hippocampal neurons, increasing the frequency and duration of kainate-induced seizures in mice [16].

HMGB1 is expressed in all cells, including neurons and glial cells. Overexpression of extracellular HMGB1 has been demonstrated in several models of neuroinflammation, including stroke and traumatic brain injury [1719]. Moreover, extracellular HMGB1 has been identified in both serum and brain tissue samples from stroke patients at 2 and $24 \mathrm{~h}$ after ischemia [12]. HMGB1 is rapidly released from dying cells after an ischemic event in the brain and later actively secreted in the subacute and chronic phase by local and infiltrating myeloid cells [1921]. HMGB1-blocking therapies have proven to be highly beneficial, providing significant neuroprotection in neuroinflammation models [17-19, 22]. Neutralising HMGB1 therapy after stroke has, however, produced conflicting findings. Administration of HMGB1 box A, a full-length HMGB1 antagonist in many inflammatory models, mediates beneficial treatment effects [18]. Additionally, therapy with an anti-HMGB1 monoclonal antibody recognising an epitope in the $\mathrm{C}$-terminal tail or short hairpin RNA-mediated HMGB1 downregulation, reduces infarction, inhibits blood-brain barrier (BBB) disruption, decreases microglia activation, and diminishes cytokine production after middle cerebral artery occlusion in rats [19, 22, 23]. However, another experimental stroke study, using a separate anti-HMGB1 monoclonal antibody (2G7) recognising an epitope in the box A domain, did not reduce the infarct volume and did not improve the neurological outcome, although the treatment alleviated sickness behaviour induced by peripheral immune responses [12].

The aim of this study was to dissect the specific contribution of HMGB1 in its various redox isoforms for the 
development of neuroinflammation in the absence of other DAMPs. We injected individual redox isoforms of HMGB1 directly into the cerebral cortex and monitored the developing neuroinflammatory response using magnetic resonance imaging (MRI) and immunohistochemistry (IHC). MRI after injection of a gadolinium-containing contrast agent is the most common non-invasive technique used clinically and in pre-clinical models to measure BBB disruption. Using MRI, we observed that both ds- and fr-HMGB1 induced BBB disruption $24 \mathrm{~h}$ after HMGB1 injection. ds- and fr-HMGB1 also promoted a local inflammatory response characterised by enhanced IL-1 $\beta$ expression, and ds-HMGB1 significantly enhanced major histocompatibility complex (MHC) class II expression and apoptosis.

\section{Materials and Methods}

\section{Preparation of Recombinant Proteins}

Endotoxin-free $(<0.003 \mathrm{EU} / \mu \mathrm{g}$ of protein $)$ recombinant HMGB1 was expressed and purified from BL21 (DE3) cells as previously described [24]. Briefly, HMGB1 DNA was subcloned into the $\mathrm{pCAL} / \mathrm{n}$ vector with a calmodulin-binding protein tag. The plasmid was transformed into Escherichia coli BL21 (DE3) cells and cultured in 2-YT media. Protein expression was induced with the addition of $1 \mathrm{mM}$ IPTG. HMGB1 was purified using calmodulin sepharose 4B resin (GE Healthcare). DNase I was added to remove any contaminating DNA, confirmed by GelRed staining of an agarose gel. Protein purity was verified by SDS-PAGE gel analysis with Coomassie Blue staining and was above $90 \%$. For the removal of contaminating endotoxin, the protein was incubated with $1 \%$ Triton $\mathrm{X} 114$ at $4^{\circ} \mathrm{C}$ for $30 \mathrm{~min}$, incubated for a further 10 $\min$ at $37^{\circ} \mathrm{C}$, and centrifuged at $18,300 \mathrm{~g}$ at $25^{\circ} \mathrm{C}$ for $10 \mathrm{~min}$. Endotoxin levels were measured using the limulus amoebocyte lysate assay at the Clinical Laboratory, Karolinska University Hospital, Stockholm, Sweden. Purified HMGB1 was in the disulfide isoform and induced cytokine production in PBMCs. To generate the fully reduced isoform, rHMGB1 was exposed to $2.5 \mathrm{mM}$ dithiothreitol (DTT) for $2 \mathrm{~h}$ at room temperature.

\section{Animals}

In vivo experiments were performed using protocols approved by the Stockholm North Ethics Committee, Sweden. Adult male Dark Agouti rats were purchased from Harlan and weighed 200$300 \mathrm{~g}$ at the time of the experiment. The animals were housed in cages of 4 at specific pathogen-free facilities at the Karolinska University Hospital (Stockholm, Sweden). Throughout the experiment the animals had free access to food and water. A 12-h light/ dark cycle was maintained at all times.

\section{Stereotaxic Surgery}

For intracerebral injection of saline (negative control), LPS, or HMGB1 the animals were anaesthetised with $4 \%$ isoflurane (maintained at $2 \%$ during the surgery) and secured into a stereotaxic frame. The scalp was swabbed with $70 \%$ ethanol, $0.2 \mathrm{~mL}$ bupiva- caine hydrochloride was applied subcutaneously, and an incision was made at the sagittal midline exposing the skull. Under microscopic guidance, a craniotomy was drilled into the cerebral cortex: $2.5 \mathrm{~mm}$ posterior and $2.5 \mathrm{~mm}$ lateral to the bregma in the right hemisphere at a depth of $2 \mathrm{~mm}$ from the brain surface. A 5- $\mu \mathrm{L}$ injection of saline, LPS $(1 \mu \mathrm{g} / \mu \mathrm{L})$, ds-HMGB1 $(1.5 \mu \mathrm{g} / \mu \mathrm{L})$, or frHMGB1 $(1.5 \mu \mathrm{g} / \mu \mathrm{L})$ diluted in PBS pH 7.2 was administered using a $10-\mu \mathrm{L}$ Hamilton syringe at a rate of $1 \mu \mathrm{L} / \mathrm{min}$. The doses of LPS and HMGB1 were chosen based on previous studies and practical considerations (e.g., the maximum amount that could be given within the injection volume before protein precipitation). The needle was left in position for a further 5 min before being slowly withdrawn. The wound was then sutured and the animals were allowed to recover.

\section{MRI Acquisition}

MRI experiments were performed $24 \mathrm{~h}$ after stereotaxic surgery in a horizontal 9.4-T magnet (Varian, Yarnton, UK). The whole procedure took between 45 and $75 \mathrm{~min}$ for each animal. A volume coil with 72-mm inner diameter and a 4-channel phased array surface coil, designed for the rat head, were employed for excitation and detection, respectively (Rapid Biomed, Würzburg, Germany). An actively tuned arterial spin labelling (ASL) coil (Rapid Biomed) was placed under the neck of the rats.

The animals were anaesthetised using $4 \%$ isoflurane and placed in a prone position in the MR-compatible bed (Rapid Biomed). Before the bed was placed into the magnet an intravenous line was placed into the tail vein for the administration of Magnevist or fluorescein isothiocyanate (FITC)-conjugated dextran to access $\mathrm{BBB}$ permeability as described later. Core body temperature was maintained at $37^{\circ} \mathrm{C}$ during scanning using a warm air system (SA Instruments, Stony Brook, NY, USA). The respiration rate was monitored (SA Instruments) and the isoflurane level was adjusted such that the respiration rate was approximately 60 inspirations per minute, i.e., in the range of $1.5-2 \%$.

The scanning protocol included fluid attenuated inversion recovery (FLAIR), T1-weighted, T2-weighted, ASL, and diffusionweighted images. A slice package of 16 contiguous slices of $0.7-\mathrm{mm}$ thickness each with a field of view of $30 \times 24 \mathrm{~mm}^{2}$ and covering the midbrain was studied with each sequence.

The FLAIR images were realised through a fast spin echo sequence preceded by an inversion pulse $(\mathrm{TR}=5 \mathrm{~s}, \mathrm{ETL}=8$, kzero $=4$, matrix $=256 \times 128, \mathrm{TI}=800 \mathrm{~ms}, 2$ averages, scan time $2 \min 50 \mathrm{~s}$ ). T1-weighted images were acquired using a spin echo sequence $(\mathrm{TR}=700 \mathrm{~ms}, \mathrm{TE}=11.86 \mathrm{~ms}$, matrix $=192 \times 128$, scan time $3 \mathrm{~min})$. ASL data were acquired using continuous ASL with a gradient echo echo-planar imaging readout module $(\mathrm{TR}=7.5 \mathrm{~s}$, $\mathrm{TE}=$ $7.12 \mathrm{~ms}$, matrix $48 \times 32$, tag duration $=3 \mathrm{~s}$, inflow time $250 \mathrm{~ms}$ ). The interleaved tagged and non-tagged images were repeated 20 times with a total scan time of $10 \mathrm{~min}$. ASL images were calculated as pixelwise (non-tagged-tagged/non-tagged) intensities. When the ASL scan was complete the rats were removed from the magnet and given a bolus of $0.5 \mathrm{~mL}$ Magnevist (contrast agent; Bayer Pharma AG, Berlin, Germany) through the tail vein.

Diffusion-weighted images were acquired using single-shot spin echo echo-planar imaging $(\mathrm{TR}=3 \mathrm{~s}, \mathrm{TE}=36.8 \mathrm{~ms}, 4$ averages, matrix $=64 \times 64$, diffusion encoding and decoding gradients of 2.6-ms duration separated by $6.9 \mathrm{~ms}$ ). The diffusion gradients were applied in 12 different directions with $40 \mathrm{Gs} / \mathrm{cm}$, resulting in $\mathrm{b}=$ $1,000 \mathrm{~mm}^{2} / \mathrm{s}$. Reference images where the diffusion gradient am- 
plitudes were set to zero were repeated twice. For each diffusionweighted image the full sequence was repeated with the inverted polarity of the readout gradient train to enable Nyquist ghost correction. The scan time for the diffusion-weighted data was 6 min $7 \mathrm{~s}$. The integrity of the BBB was assessed by repeating the FLAIR and the T1-weighted images after the completion of the diffusion-weighted images, i.e., 7 and $10 \mathrm{~min}$ after the bolus administration.

\section{Assessment of BBB Permeability using FITC-Conjugated \\ Dextran Leakage}

To further assess BBB permeability changes, a subgroup of animals were treated with $70 \mathrm{kDa}$ FITC-conjugated dextran $(1 \mathrm{~mL}$ $\mathrm{IV} ; 50 \mathrm{mg} / \mathrm{mL}$ ). The dextran was administered immediately after the MRI experiments through the tail vein and the animals were sacrificed 20 min after the injection using $\mathrm{CO}_{2}$. Sections were prepared as described below. Slides were immediately counterstained with DAPI-containing Prolong Gold anti-fade medium (Life Technologies) and viewed on a Leica TCS SP5 confocal microscope.

\section{Preparation of Brain Tissue}

The animals were sacrificed using $\mathrm{CO}_{2}$ and perfused with saline and $4 \%$ PFA. After decapitation the brains were dissected, postfixed in $4 \%$ PFA overnight and cryoprotected in $20 \%$ sucrose. Tissues were frozen using a mixture of dry ice and isopentane and stored at $-80^{\circ} \mathrm{C}$ prior to sectioning.

To ensure consistent processing of all samples the sectioning strategy was as follows: the brain was cut into three blocks and sections were cut from the middle block which contained the area where the intracerebral injection had been made. When sectioning, the start and end of the injection site was confirmed by haematoxylin and eosin staining in every animal. Coronal brain sections of $12 \mu \mathrm{M}$ were cut on a cryostat, mounted on glass slides, and dried at room temperature for $30 \mathrm{~min}$.

\section{Immunohistochemistry}

For IHC staining, PBS was used as a washing buffer. To inactivate endogenous peroxidase activity, sections were treated with $3 \%$ hydrogen peroxide diluted in PBS for 30 min. After washing, endogenous biotin was quenched using an Avidin/Biotin Blocking Kit (Vector Laboratories) according the manufacturer's instructions. Sections were washed and blocked for $1 \mathrm{~h}$ with $2 \%$ normal horse serum. Primary antibodies for IL-1 $\beta$ (R and D, AF-501-NA, $2 \mu \mathrm{g} / \mathrm{mL}$ diluted in PBS, $0.3 \%$ Triton X100) and MHC class II (OX-6; the hybridoma for OX-6, originally obtained from ATCC, was cultured and purified in our laboratory: $1 \mu \mathrm{g} / \mathrm{mL}$ diluted in PBS containing $2 \%$ normal rat serum) were added overnight at $4{ }^{\circ} \mathrm{C}$. To check for unspecific binding, sections were also stained using the appropriate isotype control antibody: goat IgG (Sigma, I5256) or mouse IgG1 (Dako, X0931). For secondary detection, biotinylated antibodies were added for $1 \mathrm{~h}$ (IL-1 $\beta$ : horse anti-goat, BA-9500, 1/1,000 and OX-6: horse anti-mouse, BA-2001, 1/1,000; Vector Laboratories). Sections were then incubated for $30 \mathrm{~min}$ with Vectastain Elite ABC reagent (Vector laboratories) followed by $\mathrm{DAB}$ solution for $2 \mathrm{~min}$. Sections were counterstained using Mayer's haematoxylin solution for $2 \mathrm{~min}$, covered with mounting medium, and viewed on a light microscope (Reichert Polyvar 2 type 302001; Leica).

\section{Immunofluorescent Staining}

To prevent non-specific binding, sections were blocked with $5 \%$ normal donkey serum for $1 \mathrm{~h}$ at room temperature. After washing with PBS, sections were incubated overnight at $4{ }^{\circ} \mathrm{C}$ with primary antibodies for CD68 (ED1; AbD Serotec, MCA341R, $0.5 \mu \mathrm{g} / \mathrm{mL}$ ) or Iba-1 (Abcam, ab107159 $1 \mu \mathrm{g} / \mathrm{mL})$ diluted in PBS, 0.3\% Triton X100. For secondary detection, Alexa Fluor ${ }^{\circledR}$ 594-conjugated donkey anti-goat antibody was added for $1 \mathrm{~h}$ (Invitrogen; A-11058, diluted $1 / 1,000$ in PBS). Immunolabelled sections were counterstained with Prolong Gold anti-fade medium with DAPI and examined on a Reichert Polyvar 2 microscope (Leica). Images were obtained on a Zeiss LSM 700 confocal microscope (Zeiss, Germany).

\section{TUNEL Staining}

Brain sections were stained using the TACS ${ }^{\circledR} 2 \mathrm{TdT}$ in situ DAB kit (Tevigen) according to the manufacturer's instructions. Sections were counterstained with methyl green and examined on a Reichert Polyvar 2 type 302001 microscope.

\section{MRI Analysis}

Volumetric measurements were performed on gadolinium-enhanced MRI data using OsiriX imaging software (OsiriX Foundation, Geneva, Switzerland) [25]. Volumes were approximated by manual tracing of the gadolinium-enhanced $\mathrm{T} 1$ lesion perimeter. Manual tracing was performed in section intervals of $1 \mathrm{~mm}$; the final volume was established through software interpolation and volume calculation. Volumetric measurements were also calculated for the gadolinium-enhanced FLAIR images. MRI analysis was performed by an independent assessor blinded to the treatment groups.

\section{Evaluation of IHC and Immunofluorescent Staining}

Expression of MHC II was analyzed on a Polyvar II microscope (Leica Microsystems, Wetzlar, Germany) by an assessor blinded to the treatment groups. The percentage of positive staining in the ipsilateral hemisphere was estimated and assigned a score on the scale of $1-3$ : 1 , less than $1 \% ; 2,10-20 \% ; 3$, more than $20 \% \mathrm{MHC}+$ cells present along the injection site.

Quantitative analysis was performed for IL-1 $\beta$, CD68, and TUNEL staining. Sections were analyzed on a Leica DM RXA2 microscope using Leica QWin V3 software (Leica Microsystems). The number of positive cells was counted in 12 fields along the injection trajectory using a $200 \times$ magnification and expressed as a percentage of the total cells (For IHC staining the total cells were counted as haematoxylin+ and for immunofluorescent staining as DAPI+). In addition, for the CD68 staining the total area in the ipsilateral hemisphere containing positive cells was measured using the Leica QWin V3 software.

\section{ELISA}

Serum $\alpha_{1}$-acid glycoprotein (AGP) concentrations were determined by ELISA according to the manufacturer's instructions (Life Diagnostics Ltd, UK).

\section{Statistical Analysis}

All statistical analyses was performed using GraphPad Prism, V6.01. Data are expressed as median \pm interquartile range (IQR). The data were first analyzed for normality. $p$ values were calculated using a non-parametric Kruskal-Wallis test with Dunn's multiple comparison correction. Differences were considered to be significant when $p \leq 0.05$. 


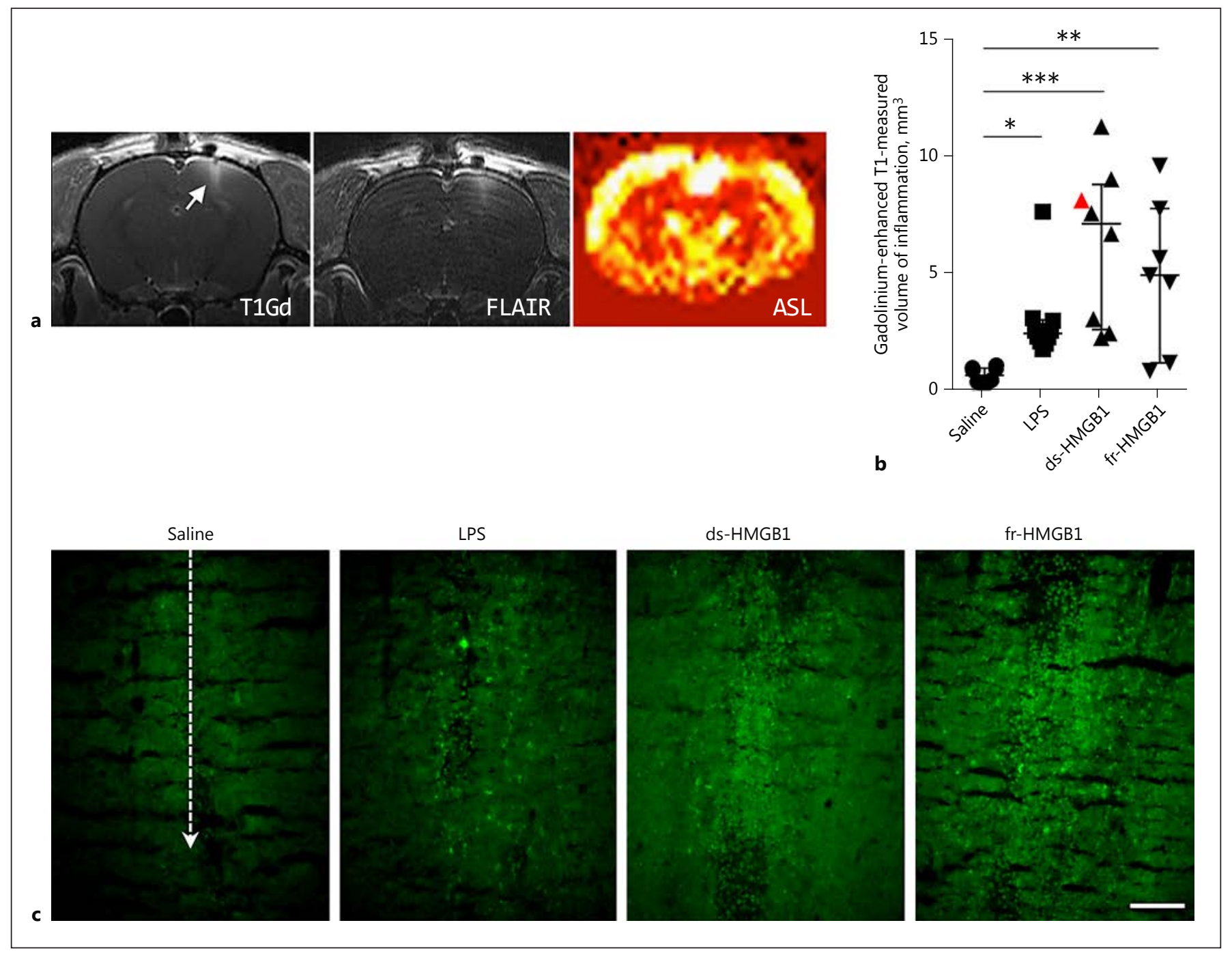

Fig. 1. Intracerebral injection of HMGB1 induces blood-brain barrier leakage and neuroinflammation. HMGB1, high-mobility group box 1; LPS, lipopolysaccharide; ds, disulfide; fr, fully reduced. a Representative T1-weighted, FLAIR, and ASL images (non-tagged-tagged/non-tagged) collected after ds-HMGB1 injection (animal identified by a red triangle in $\mathbf{b}$ ). T1-weighted and FLAIR images were collected after gadolinium. b Quantification of the volume of inflammation in gadolinium-enhanced T1weighted images. LPS, ds-HMGB1, and fr-HMGB1 significantly

\section{Results}

A total of 34 animals were included in the study; saline $(n=8)$, LPS $(n=10)$, ds-HMGB1 $(n=8)$, and fr-HMGB1 $(n=8)$. One animal, treated with fr-HMGB1, was excluded from the MRI analysis due to technical problems with the intravenous line, but was included in the IHC and immunofluorescence studies. increased gadolinium uptake compared to the saline controls (saline vs. LPS, $p=0.0413$; saline vs. ds-HMGB1, $p=0.0002$; saline vs. fr-HMGB1, $p=0.0076)$. Data are expressed as median \pm IQR. $p$ values were calculated using a Kruskal-Wallis test corrected for multiple comparisons using the Dunn test ( $n=7-10$ rats/group). ${ }^{*} p \leq 0.05,{ }^{* *} p \leq 0.01,{ }^{* * *} p \leq 0.001$. c Representative images showing extravasation of $70 \mathrm{kDa}$ FITC-conjugated dextran. Dashed line indicates the location of the injection site ( $n=3-4$ rats/group). Scale bar, $100 \mu \mathrm{m}$.

The BBB is open $24 \mathrm{~h}$ after reperfusion injury and again after 3-4 days. In this study we chose to investigate the 24-h time point as we wanted to characterise the direct effects of HMGB1, which has a relatively short half-life, and not those triggered by the activation of subsequent downstream signalling cascades. We decided to perform the experiment in only one gender to maximise the number of animals we could include in 
each group. We chose to use male rats as they have been used previously in studies of neuroinflammation and stroke.

\section{MRI and Immunofluorescence Studies to Investigate HMGB1-Induced BBB Damage}

MRI studies were performed to investigate the effect of extracellular intracerebral HMGB1 on BBB permeability and brain oedema formation. Representative images from one animal are shown in Figure 1a. Increased signals, indicative of BBB damage, were detected in the gadolinium-enhanced T1-weighted images and volumes were quantified by manual tracing. Animals treated with LPS $\left(2.5 \pm 0.9 \mathrm{~mm}^{3}\right)$, ds-HMGB1 $\left(7.2 \pm 6.2 \mathrm{~mm}^{3}\right)$, and frHMGB1 $\left(5.0 \pm 6.0 \mathrm{~mm}^{3}\right)$ had significantly increased gadolinium extravasation, a marker for BBB damage, compared to the control group $\left(0.7 \pm 0.6 \mathrm{~mm}^{3}\right.$; Fig. $\left.1 \mathrm{~b}\right)$. We did not detect any statistical differences between the animals injected with ds- or fr-HMGB1 and LPS. A strong agreement of high signal in the FLAIR sequence with the volumes manually traced in the gadolinium-enhanced T1-weighted images was observed. These findings further strengthened the indications of ongoing inflammation in the area of BBB damage. The results could also be identified in the T2-weighted images (data not shown). Additional data were collected from ASL and diffusion experiments; however, no significant changes were detected in these images at the 24-h time point (data not shown).

A subgroup of animals $(n=19)$ received an intravenous injection of $70 \mathrm{kDa}$ FITC-conjugated dextran after the MRI imaging to further evaluate the BBB permeability changes observed by MRI. Consistent with the MRI results, increased FITC dextran extravasation was observed along the injection site following exposure to ds- or fr-HMGB1 compared to the vehicle control (Fig. 1c). Increased extravasation of FITC-conjugated dextran was also apparent after LPS injection, although the intensity of the staining was much lower compared to the HMGB1-treated animals.

Taken together, the results from the MRI and immunofluorescence studies demonstrated that intracerebral injection of ds- and fr-HMGB1 increased BBB permeability via a redox-independent mechanism.

\section{Characterisation of the Inflammatory Response}

Induced by Intracerebral Injection of HMGB1

To analyze the inflammatory response induced by HMGB1, sections from a subgroup of animals $(n=16)$ were stained with an antibody recognising the Iba-1 protein, which is expressed on both resting and activated monocytes and microglia cells. Iba- $1+$ cells in the contra- lateral hemisphere expressed a ramified morphology consistent with a resting state (data not shown). In contrast, the majority of Iba-1+ cells located directly along the injection site in all of the groups expressed a rounded or activated morphology with only a few ramified cells (online suppl. Fig. S1; for all online suppl. material, see www.karger.com/doi/10.1159/000487056).

To quantify the number of activated cells, sections were stained with a monoclonal antibody (ED1) binding to the CD68 antigen, which is a molecule upregulated on phagocytic macrophages and microglia but not resting cells. To start, we quantified the number of CD68+ cells present directly at the injection site (Fig. 2a, b). CD68+ cells were detected in all of the fields studied in all animals. No significant differences were detected between the groups, although there was a slight trend towards a higher number of phagocytic cells in the HMGB1-injected animals.

We observed that CD68-expressing cells were also present in regions outside the injection site in the cerebral cortex and we subsequently measured the total area containing positive cells in the ipsilateral hemisphere (Fig. $2 \mathrm{~d}$ ). On average, $5.6 \pm 3.4 \%$ of the tissue stained positive for CD68+ cells in the saline group. In the animals treated with ds- or fr-HMGB1 there was a tendency towards higher CD68 protein levels with $10.6 \pm 2.8$ and $12.5 \pm 9.0 \%$ of the tissue staining positive, respectively. In contrast, there was a statistically significant increase in CD68 expression in the LPS-exposed animals (13.8 \pm $8.0 \%, p=0.01)$.

MHC class II synthesis and cell surface expression can be upregulated in response to inflammatory stimuli. We used the OX-6 antibody to evaluate MHC class II protein levels in the CNS after injections of saline, LPS, or HMGB1. Only ds-HMGB1 $(2.0 \pm 1.0)$, but not LPS $(2 \pm 2)$ or fr-HMGB1 (1.5 \pm 1.75$)$, significantly increased MHC class II levels compared to the saline-injected group (1.0 \pm 0 ; Fig. 3a, b).

Previous studies have established that ds-HMGB1 induces cytokine production via TLR4/MD2 signalling [4]. Sections were stained using an antibody against IL-1 $\beta$ to examine proinflammatory cytokine production in the CNS (Fig. 4a). IL-1 $\beta$ levels were significantly increased after cerebral injection of LPS $(8.9 \pm 11.7 \%)$, ds-(12.1 \pm $9.1 \%)$, or fr-HMGB1 $(6.7 \pm 9.8 \%)$ compared to the control rats $(1.8 \pm 3.0 \%)$; (Fig. $4 \mathrm{~b})$. IL- $1 \beta+$ cells were predominantly located in the area directly along the injection trajectory. A few scattered cells appeared in the ipsilateral hemisphere, while no positive cells were observed in the contralateral hemisphere. 

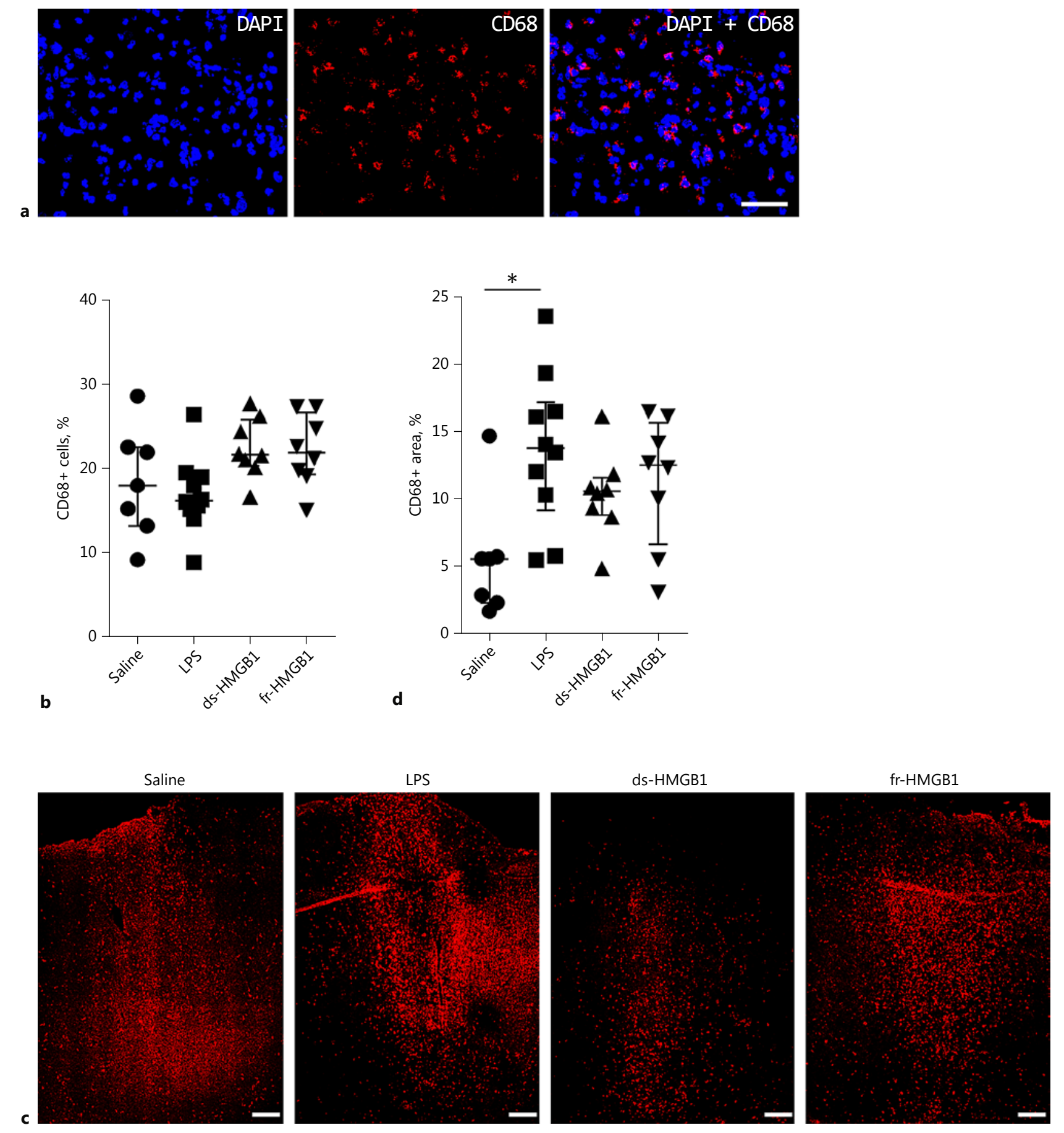

Fig. 2. CD68 protein levels are increased in response to cerebral LPS but not HMGB1. Representative sections showing the level of CD68 protein present at the injection site and in the ipsilateral hemisphere. See legend to Fig. 1 for abbreviations. a High-magnification images showing CD68+ staining at the injection site. Scale bar, $25 \mu \mathrm{m}$. b Quantification of CD68+ cells at the injection site. No significant differences were detected between the groups. c Low magnification image illustrating the distribution of CD68+ cells in the cerebral cortex of the ipsilateral hemisphere. Scale bar, $200 \mu \mathrm{m}$. d Total area positive for CD68 cells in the ipsilateral hemisphere. Rats injected with LPS had increased CD68 protein levels compared to the saline controls $(p=0.0116)$. The difference in the HMGB1-treated animals was not significant. Data are expressed as median \pm IQR. $p$ values were calculated using a Kruskal-Wallis test corrected for multiple comparisons using the Dunn test $(n=8-10$ rats/group). ${ }^{*} p \leq 0.05,{ }^{* *} p \leq 0.01,{ }^{* * *} p \leq 0.001$. 


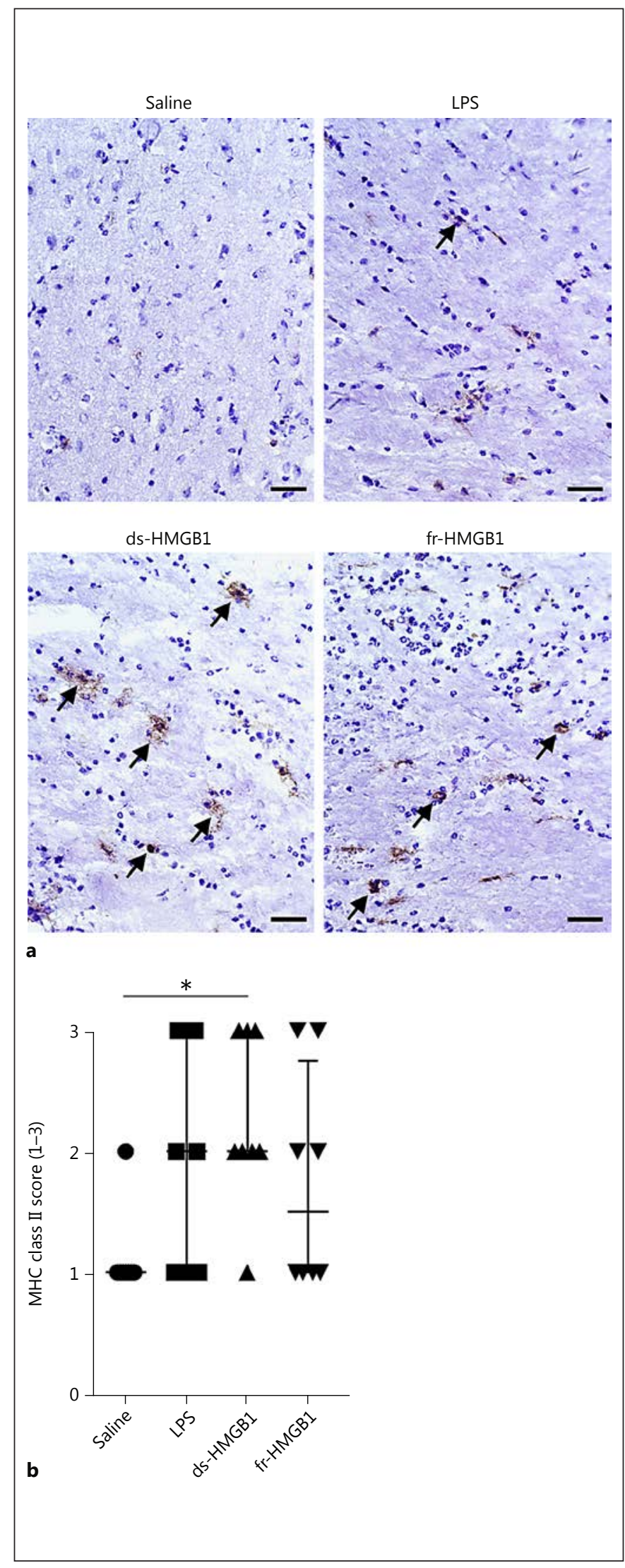

Taken together, the results demonstrated that dsHMGB1 induced inflammation, characterised by increased MHC class II expression and cytokine production $24 \mathrm{~h}$ after the injection. fr-HMGB1 also generated inflammation reflected by IL- $1 \beta$ but not significant MHC class II production.

\section{ds-HMGB1-Induced Apoptosis in the CNS}

DNA fragmentation was measured using the TUNEL staining assay to investigate possible cytotoxic effects induced by HMGB1 (Fig. 5a). Scattered apoptotic cells were detected along the injection pathway in the saline-injected animals $(1.7 \pm 1.1 \%)$. Intracerebral injection of dsHMGB1 induced a 7 -fold increase in the number of TUNEL+ cells $(12.2 \pm 13.8 \%, p=0.01$; Fig. $5 b)$. The cells were observed directly along the injection trajectory and were not detected outside the cortex or in the contralateral hemisphere. A non-significant increase was observed in the animals receiving LPS $(1.8 \pm 9.4 \%, p=>0.99)$ or fr-HMGB1 $(6.2 \pm 12.0 \%, p=0.16)$.

\section{A Single Intracerebral Injection of LPS Caused a}

Systemic Immune Response in Contrast to HMGB1

Serum levels of the human and rat acute phase reactant AGP (orosomucoid) increase during inflammation [26]. To examine the systemic immune response to intracerebral injections of saline, LPS, or HMGB1, serum AGP concentrations were measured by ELISA. Serum AGP levels after injections of saline, ds-HMGB1, or fr-HMGB1 were similar to pre-injection control samples (118.7 \pm $117.6,135.0 \pm 63.2$, or $166.9 \pm 274.5 \mu \mathrm{g} / \mathrm{mL}$ vs. $105.3 \pm$ $25.0 \mu \mathrm{g} / \mathrm{mL}$, respectively; Fig. 6). In contrast, LPS-injected animals expressed significantly increased serum AGP concentrations $(322.1 \pm 377.0 \mu \mathrm{g} / \mathrm{mL}, p=0.003)$.

\section{Discussion}

The release of HMGB1 from stressed and dying brain cells is an early event occurring after a trauma. It is becoming increasingly clear that different types of stressors

Fig. 3. ds-HMGB1 upregulates major histocompatibility complex (MHC) class II expression in the CNS. a Representative MHC class II staining in coronal brain sections. b Scoring of MHC class II levels in the ipsilateral hemisphere. Scale bar, $50 \mu \mathrm{m}$. Data are expressed as median \pm IQR. $p$ values were calculated using a KruskalWallis test corrected for multiple comparisons using the Dunn test ( $n=8-10$ rats/group). ${ }^{*} p \leq 0.05,{ }^{* *} p \leq 0.01,{ }^{* * *} p \leq 0.001$. See legend to Fig. 1 for abbreviations. 
and cell death modes release different redox isoforms of HMGB1 into the extracellular environment [27]. Recently, it has been shown that post-translational oxidative modifications of the three cysteine residues present in HMGB1 generate different redox isoforms that have the ability to interact with multiple diverse receptors, mediating separate inflammatory functions. In the present study we aimed to dissect the contribution of extracellular, cerebral HMGB1, in its disulfide or fully reduced redox form, to neuroinflammation. We did not include oxHMGB1 as to date this redox form has not been found to mediate any pro-inflammatory function in vivo $[4,6]$. Our results clearly demonstrate that ds- and fr-HMGB1 function as pro-inflammatory mediators in the CNS, promoting BBB disruption and cytokine production. Additionally, ds-HMGB1 activates antigen-presenting cells and mediates cytotoxicity.

The BBB, the physical interface that restricts the movement of plasma components including neurotoxic substances between the blood and CNS, may become leaky during inflammation. Accumulating evidence indicates that BBB dysfunction is an important pathogenic mechanism in several neuroinflammatory and neurodegenerative diseases where extracellular HMGB1 levels are known to be elevated. By the use of MRI and immunofluorescence experiments our study clearly demonstrates that extracellular HMGB1 present in brain tissue mediates BBB disruption. The comprised permeability to blood borne substances was reflected by significantly enhanced extravasation of gadolinium (Fig. 1b) and FITC-conjugated dextran (Fig. 1c) in both groups of HMGB1-injected animals compared to the saline controls. Moreover, ds- and fr-HMGB1 induced a similar degree of BBB disruption, suggesting that the mechanisms regulating HMGB1-induced BBB modifications occur independently of the redox status of the cysteine residues, which have been shown to regulate multiple HMGB1-induced inflammatory functions $[4,8,24]$.

Fig. 4. Intracerebral extracellular HMGB1 and LPS induce proinflammatory cytokine production. a Representative sections stained using an anti-IL- $1 \beta$ antibody. Arrows indicate examples of positive cells. Scale bar, $50 \mu \mathrm{m}$. b Quantification of IL-1 $\beta$-expressing cells along the injection site. Positive cells were IL- $1 \beta+/$ haematoxylin+. Cytokine levels were significantly increased in rats treated with LPS, ds-HMGB1, or fr-HMGB1 compared to the saline controls ( $p=0.0271,0.0055$, and 0.0293, respectively). Data are expressed as median \pm IQR. $p$ values were calculated using a Kruskal-Wallis test corrected for multiple comparisons using the Dunn test $(n=$ $8-10$ rats/group). ${ }^{*} p \leq 0.05,{ }^{* *} p \leq 0.01,{ }^{* * *} p \leq 0.001$. See legend to Fig. 1 for abbreviations.

Extracellular HMGB1 Initiates CNS Inflammation

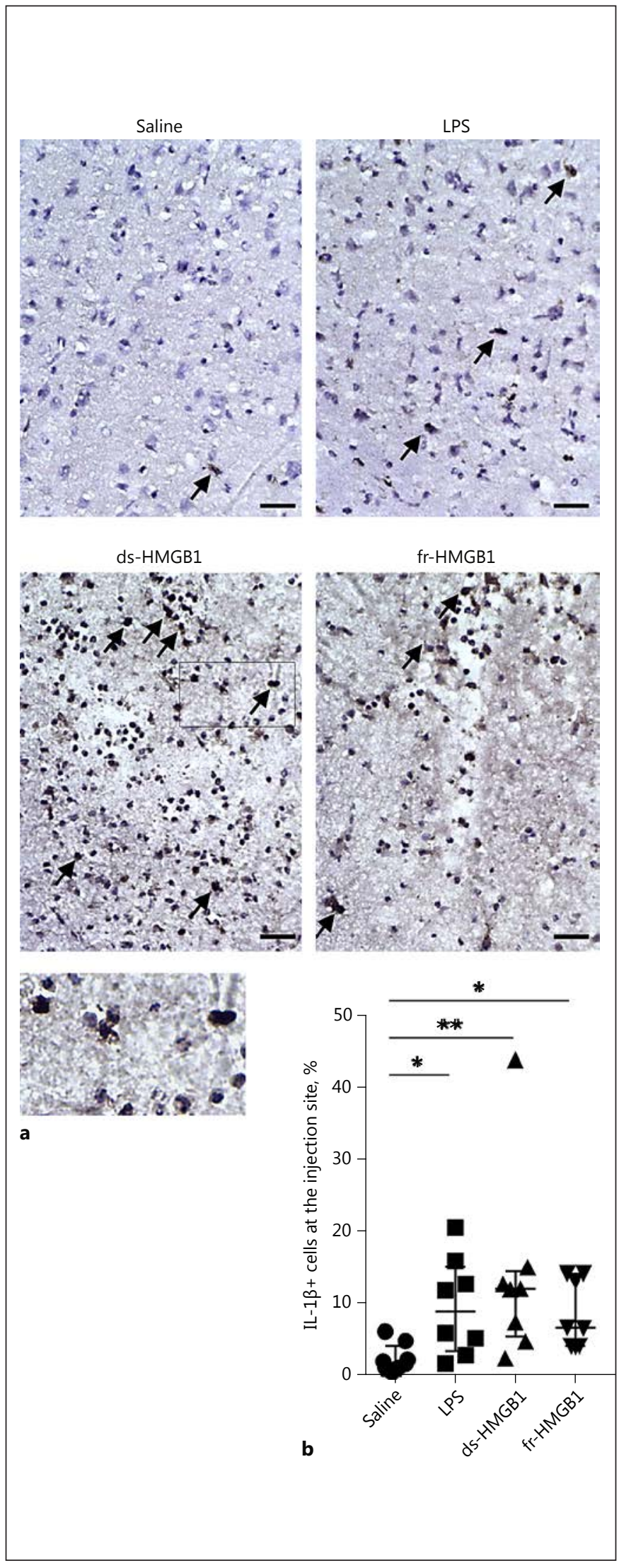

J Innate Immun

DOI: $10.1159 / 000487056$ 


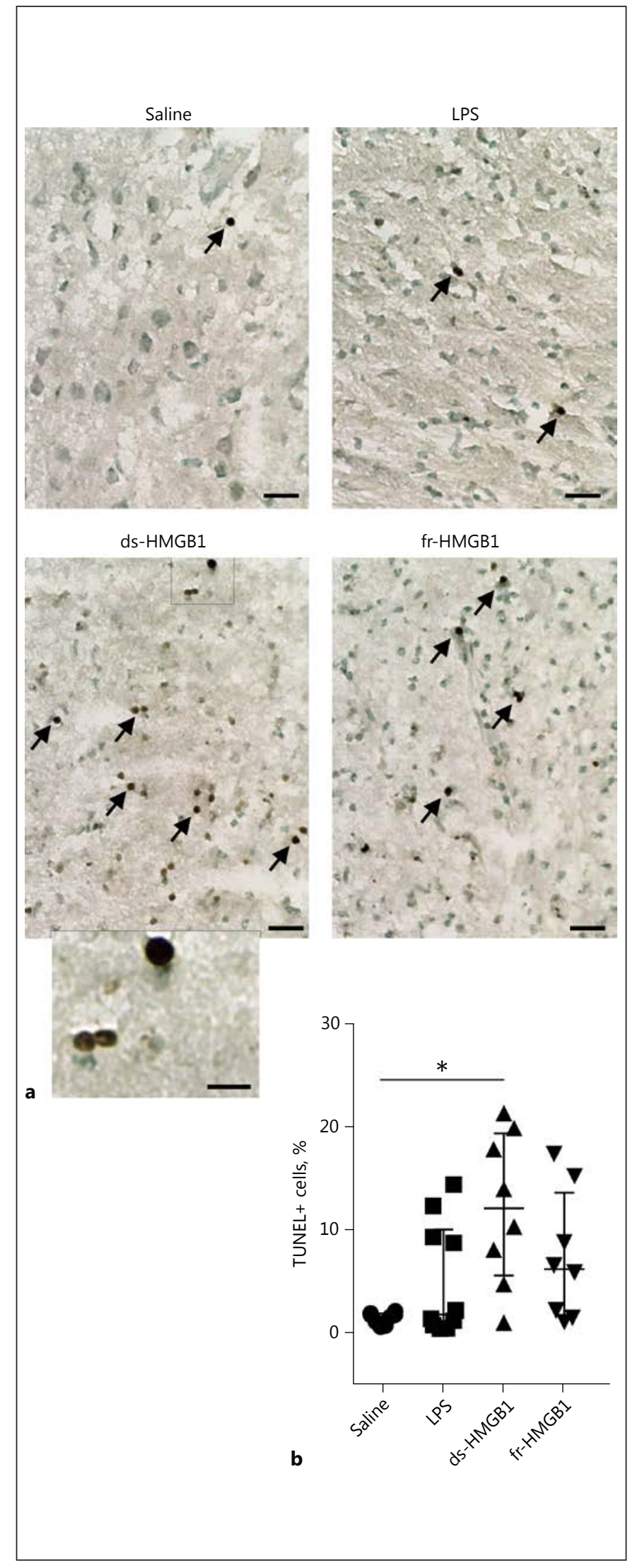

Protection against BBB disruption in ischemic and neurodegenerative models using monoclonal antiHMGB1 antibodies neutralising all redox isoforms has previously been reported $[17,28]$. In addition, Zhang et al. [22] have demonstrated that HMGB1 with no specified redox state acts directly on vascular endothelial cells and pericytes to induce morphological changes, thus increasing BBB permeability.

CD68 is an activation marker for monocytes and tissue-resident macrophages, such as microglia cells. Immunofluorescence studies confirmed that CD68+ cells were present at the injection site in all animals including the saline controls, indicating that the injection alone induced some microglia/monocyte activation (Fig. 2b). We did not detect changes in the other markers of inflammation or increased BBB disruption in the control group. There was a tendency towards a higher number of positive cells in both HMGB1 groups; however, this did not reach statistical significance. Microglia cells can become primed by ageing, traumatic brain injury, or in neurodegenerative diseases [29]. It is possible that microglia cells primed by the injection may have exhibited an enhanced response to the HMGB1 isoforms. Extracellular release of HMGB1 occurs after cell death or cell activation caused by an injury/trauma, thus it could be expected that some cells will be primed when encountering HMGB1 in vivo.

Apart from modifying BBB integrity, ds-HMGB1 also induced a local inflammatory response characterised by significantly increased MHC class II expression (Fig. $3 b$ ), IL-1 $\beta$ production (Fig. $4 b$ ), and cytotoxicity (Fig. 5b). fr-HMGB1 promoted a similar increase in IL$1 \beta$ to ds-HMGB1 but did not significantly upregulate MHC class II expression or apoptosis, possibly suggesting that these effects are a consequence of HMGB1TLR4 interactions, which require an intramolecular disulfide bond and a reduced $\mathrm{C} 106$ residue for ligation and signalling $[4,24]$. TLR4 has been proposed to have a

Fig. 5. Extracellular intracerebral ds-HMGB1 induces cell death. a Representative sections stained for DNA fragmentation using the TUNEL assay. Arrows indicate examples of positive cells. Scale bar, $50 \mu \mathrm{m}(20 \mu \mathrm{m}$ in the high-magnification image). b Percentage of TUNEL+ cells present along the injection site in each animal. Rats injected with ds-HMGB1 had significantly more TUNEL+ cells present at the injection site ( $p=0.01$ vs. saline). Data are expressed as median \pm IQR. $p$ values were calculated using a KruskalWallis corrected for multiple comparisons using the Dunn test ( $n=8-10$ rats/group). ${ }^{*} p \leq 0.05,{ }^{* *} p \leq 0.01,{ }^{* * *} p \leq 0.001$. See legend to Fig. 1 for abbreviations. 
pathogenic function in several models of neuroinflammation $[30,31]$. Upregulation of IL- $1 \beta$ mRNA expression by HMGB1 during ethanol-induced neuro-immune signalling is TLR4 dependent and is reduced in the presence of specific receptor antagonists or using TLR4-specific siRNA [11]. Cerebroventricular dsHMGB1 and to a lesser extent fr-HMGB1 also increase TNF- $\alpha$ production [32]. Recently, Frank et al. [33] found that ds-HMGB1 injected into the cisterna magna increases NF- $\kappa$ BI $\alpha$ mRNA, NLRP 3 mRNA, and IL- $1 \beta$ protein expression in the hippocampus within $2 \mathrm{~h}$. In contrast to our study, no cytokine production was detected after fr-HMGB1 exposure; this may be due to differences in the redox state in different brain regions which affect the modification of the protein in vivo. IL- $1 \beta$ has been previously established to exert a central role in the inflammatory response to trauma in the CNS. Holmin and Mathiesen [34] demonstrated that cerebral IL-1 $\beta$ causes a widespread inflammatory response characterised by infiltration of inflammatory cells, apoptosis of resident brain cells along with infiltrating cells, BBB

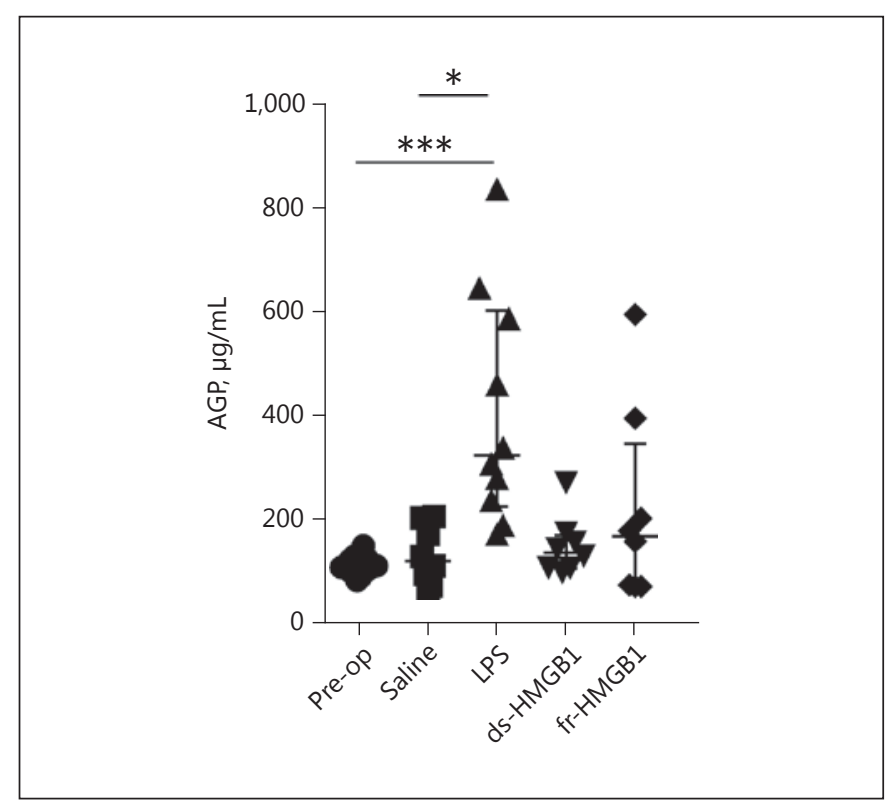

Fig. 6. Intracerebral LPS induces systemic inflammation serum $\alpha_{1^{-}}$ acid glycoprotein (AGP) concentrations, as determined by ELISA. Significantly increased AGP levels were detected in LPS-treated animals, but not in HMGB1-treated animals (LPS vs. pre-op, $p=$ 0.003 ; LPS vs. saline, $p=0.0129$ ). Data are expressed as median \pm IQR. $p$ values were calculated using a Kruskal-Wallis test corrected for multiple comparisons using the Dunn test $(n=8-12$ rats/ group). ${ }^{*} p \leq 0.05,{ }^{* *} p \leq 0.01,{ }^{* * *} p \leq 0.001$. See legend to Fig. 1 for abbreviations.

Extracellular HMGB1 Initiates CNS Inflammation leakage, and brain oedema. Moreover, mice lacking IL-1 experience reduced brain damage after middle cerebral artery occlusion [35], and IL-1 inhibition using recombinant IL-1 receptor antagonist or an anti-IL-1 $\beta$ antibody protects against ischemic injury [36].

The degree of BBB disruption and inflammation varied between animals within the HMGB1-treated groups. It is likely that this variation was caused by modification of the redox status of the recombinant HMGB1 after the injection was performed, induced by the oxidative environment in the extracellular brain tissue. fr- and dsHMGB1 are interconvertible, while terminal oxidation of the cysteine residues to sulphonyl groups is an irreversible process. Thus, fr-HMGB1 may be susceptible to ROS-mediated oxidation and conversion to ds-HMGB1 in vivo. Indeed, fr-HMGB1 has a relatively short half-life ( $\sim 17 \mathrm{~min})$ in biological fluids such as serum and saliva [37]. Other studies support this hypothesis and our findings are in agreement with these investigations $[6,16,32$, 38]. Lian et al. [32] observed similar cytokine production and depressive-like behaviour after intracerebroventricular injection of either ds- or fr-HMGB1, and these effects were mediated via TLR4 and not RAGE or CXCL12, the receptors for fr-HMGB1. Furthermore, Balosso et al. [16] reported that ds- and fr-HMGB1 enhanced NMAD-induced $\mathrm{Ca}^{2+}$ influx equally well, and the frequency and duration of kainic acid induced seizures in mice. These effects were not observed with an HMGB1 mutant protein resistant to oxidation and additionally, were inhibited using TLR4-specific antagonists. Moreover, Venereau et al. $[6,38]$ have proposed that fr-HMGB1 released from necrotic cells can be oxidised to ds-HMGB1 extracellularly, and together with ds-HMGB1 released from infiltrating leukocytes enhances the pro-inflammatory response after peripheral tissue injury.

Previous studies have found that increased levels of HMGB1 also induce behavioural changes. Agalave et al. [15] found that ds-HMGB1, but not the fully reduced or oxidised isoforms, mediates TLR4-dependent mechanical hypersensitivity. In addition, intracerebroventricular ds- and fr-HMGB1 induce depressive behaviour [32], and elevated HMGB1 levels are associated with the development of systemic sickness syndrome and cognitive dysfunction [12].

Emerging evidence indicates that HMGB1 may act as a mediator that links systemic and CNS immune responses. It has recently been shown that the HMGB1RAGE axis is involved in modulating post-brain injury systemic immune alterations in trauma and post-operative patients [12]. These changes are characterised by 
an initial over-activation of immune responses followed by immune suppression and increased susceptibility to infections. In addition, increased systemic HMGB1 levels following surgery or critical illness have been associated with cognitive dysfunction in experimental studies [39]. To test whether HMGB1 injected intracerebrally initiated alterations in the production of systemic inflammatory mediators in our study, we measured levels of the acute phase protein AGP which is produced in the liver in response to inflammation. No changes were detected in serum AGP levels in the animals injected with ds- or fr-HMGB1 compared to the control group; however, LPS caused more than a 3-fold increase in AGP serum levels (Fig. 6). It is interesting to note that HMGB1 had a tendency to induce a higher BBB leakage than LPS, while LPS induced a systemic inflammatory response not observed in the HMGB1-exposed group. However, it is difficult to compare the AGP levels in the LPS and HMGB1 groups since the time course of AGP release is likely to vary between the treatments. HMGB1 is a late mediator of LPS-induced inflammation, released more than $8 \mathrm{~h}$ after LPS treatment [3], and thus direct injection of HMGB1 may have caused peak AGP elevations at an earlier time point not investigated in these experiments. It is well known that systemic LPS can activate local inflammation in the CNS, including activation of microglia and TNF production [40]. Our results confirm that cerebral LPS also activates systemic inflammation.

\section{Conclusions}

The results from this study demonstrate that HMGB1 initiates inflammation in the CNS, inducing BBB disruption and a local inflammatory response. Our findings suggest that fr-HMGB1 released from dying cells in response to a trauma induces significant BBB leakage, potentially allowing an influx of circulating cells. In the extracellular environment fr-HMGB1 may be converted to ds-HMGB1, an isoform also released from activated cells to induce proinflammatory cytokine production, upregulation of antigen-presenting molecules, and apoptosis. Thus, this study delineates the contribution of the different HMGB1 redox isoforms to the inflammatory process resulting from tissue trauma. It also indicates that HMGB1 blockade might be beneficial in conditions involving exaggerated neuroinflammation such as after stroke and CNS trauma.

\section{Acknowledgements}

The authors would like to thank the staff at the Department of Comparative Medicine, Karolinska Institutet, in particular Kicki Edwardsson, for technique assistance. This work was supported by grants from the Söderberg Foundation, the Swedish Heart and Lung Association, The Linné Center CERIC, Karolinska Institutet, and Stockholm County Council (SLL).

\section{Disclosure Statement}

The authors declare that they have no competing interests.

\section{References}

$>1$ Gelderblom M, Sobey CG, Kleinschnitz C, Magnus T: Danger signals in stroke. Ageing Res Rev 2015;24:77-82.

72 Thundyil J, Lim KL: DAMPs and neurodegeneration. Ageing Res Rev 2015;24:17-28.

>3 Wang H, Bloom O, Zhang M, Vishnubhakat JM, Ombrellino M, Che J, Frazier A, Yang H, Ivanova S, Borovikova L, Manogue KR, Faist E, Abraham E, Andersson J, Andersson U, Molina PE, Abumrad NN, Sama A, Tracey KJ: HMG-1 as a late mediator of endotoxin lethality in mice. Science 1999;285:248-251.

4 Yang H, Lundback P, Ottosson L, Erlandsson-Harris H, Venereau E, Bianchi ME, AlAbed Y, Andersson U, Tracey KJ, Antoine DJ: Redox modification of cysteine residues regulates the cytokine activity of high mobility group box-1 (HMGB1). Mol Med 2012;18: 250-259.

5 Hori O, Brett J, Slattery T, Cao R, Zhang J, Chen JX, Nagashima M, Lundh ER, Vijay S, Nitecki D, et al: The receptor for advanced glycation end products (RAGE) is a cellular binding site for amphoterin. Mediation of neurite outgrowth and co-expression of rage and amphoterin in the developing nervous system. J Biol Chem 1995;270:25752-25761.

6 Venereau E, Casalgrandi M, Schiraldi M, Antoine DJ, Cattaneo A, De Marchis F, Liu J, Antonelli A, Preti A, Raeli L, Shams SS, Yang H, Varani L, Andersson U, Tracey KJ, Bachi A, Uguccioni M, Bianchi ME: Mutually exclusive redox forms of HMGB1 promote cell recruitment or proinflammatory cytokine release. J Exp Med 2012;209:1519-1528.

7 Hreggvidsdottir HS, Ostberg T, Wahamaa H, Schierbeck H, Aveberger AC, Klevenvall L, Palmblad K, Ottosson L, Andersson U, Harris HE: The alarmin HMGB1 acts in synergy with endogenous and exogenous danger signals to promote inflammation. J Leukoc Biol 2009; 86:655-662.

8 Schiraldi M, Raucci A, Munoz LM, Livoti E, Celona B, Venereau E, Apuzzo T, De Marchis
F, Pedotti M, Bachi A, Thelen M, Varani L, Mellado M, Proudfoot A, Bianchi ME, Uguccioni M: HMGB1 promotes recruitment of inflammatory cells to damaged tissues by forming a complex with CXCL12 and signaling via CXCR4. J Exp Med 2012;209:551-563.

$\checkmark 9$ O'Connor KA, Hansen MK, Rachal Pugh C, Deak MM, Biedenkapp JC, Milligan ED, Johnson JD, Wang H, Maier SF, Tracey KJ, Watkins LR: Further characterization of high mobility group box 1 (HMGB1) as a proinflammatory cytokine: central nervous system effects. Cytokine 2003;24:254-265.

10 Agnello D, Wang H, Yang H, Tracey KJ, Ghezzi P: HMGB-1, a DNA-binding protein with cytokine activity, induces brain TNF and IL-6 production, and mediates anorexia and taste aversion. Cytokine 2002;18:231-236.

11 Zou JY, Crews FT: Release of neuronal HMGB1 by ethanol through decreased HDAC activity activates brain neuroimmune signaling. PLoS One 2014;9:e87915. 
-12 Liesz A, Dalpke A, Mracsko E, Antoine DJ, Roth S, Zhou W, Yang H, Na SY, Akhisaroglu M, Fleming T, Eigenbrod T, Nawroth PP, Tracey KJ, Veltkamp R: DAMP signaling is a key pathway inducing immune modulation after brain injury. J Neurosci 2015;35:583598.

-13 Weber MD, Frank MG, Tracey KJ, Watkins LR, Maier SF: Stress induces the danger-associated molecular pattern HMGB-1 in the hippocampus of male Sprague Dawley rats: a priming stimulus of microglia and the NLRP3 inflammasome. J Neurosci 2015;35:316-324.

-14 Yamasoba D, Tsubota M, Domoto R, Sekiguchi F, Nishikawa H, Liu K, Nishibori M, Ishikura H, Yamamoto T, Taga A, Kawabata A: Peripheral HMGB1-induced hyperalgesia in mice: redox state-dependent distinct roles of RAGE and TLR4. J Pharmacol Sci 2016;130: 139-142.

15 Agalave NM, Larsson M, Abdelmoaty S, Su J, Baharpoor A, Lundback P, Palmblad K, Andersson U, Harris H, Svensson CI: Spinal HMGB1 induces TLR4-mediated long-lasting hypersensitivity and glial activation and regulates pain-like behavior in experimental arthritis. Pain 2014;155:1802-1813.

16 Balosso S, Liu J, Bianchi ME, Vezzani A: Disulfide-containing high mobility group box-1 promotes $\mathrm{N}$-methyl-D-aspartate receptor function and excitotoxicity by activating Tolllike receptor 4-dependent signaling in hippocampal neurons. Antioxid Redox Signal 2014; 21:1726-1740.

17 Okuma Y, Liu K, Wake H, Zhang J, Maruo T, Date I, Yoshino T, Ohtsuka A, Otani N, Tomura S, Shima K, Yamamoto Y, Yamamoto H, Takahashi HK, Mori S, Nishibori M: Antihigh mobility group box-1 antibody therapy for traumatic brain injury. Ann Neurol 2012; 72:373-384.

18 Muhammad S, Barakat W, Stoyanov S, Murikinati S, Yang H, Tracey KJ, Bendszus $M$, Rossetti G, Nawroth PP, Bierhaus A, Schwaninger M: The HMGB1 receptor RAGE mediates ischemic brain damage. J Neurosci 2008; 28:12023-12031.

-19 Kim JB, Sig Choi J, Yu YM, Nam K, Piao CS, Kim SW, Lee MH, Han PL, Park JS, Lee JK: HMGB1, a novel cytokine-like mediator linking acute neuronal death and delayed neuroinflammation in the postischemic brain. J Neurosci 2006;26:6413-6421.
20 Singh V, Roth S, Veltkamp R, Liesz A: HMGB1 as a key mediator of immune mechanisms in ischemic stroke. Antioxid Redox Signal 2016;24:635-651.

21 Kim JB, Lim CM, Yu YM, Lee JK: Induction and subcellular localization of high-mobility group box-1 (HMGB1) in the postischemic rat brain. J Neurosci Res 2008;86:1125-1131.

22 Zhang J, Takahashi HK, Liu K, Wake H, Liu R, Maruo T, Date I, Yoshino T, Ohtsuka A, Mori S, Nishibori M: Anti-high mobility group box-1 monoclonal antibody protects the blood-brain barrier from ischemia-induced disruption in rats. Stroke 2011;42: 1420-1428.

23 Liu K, Mori S, Takahashi HK, Tomono Y, Wake H, Kanke T, Sato Y, Hiraga N, Adachi $\mathrm{N}$, Yoshino T, Nishibori M: Anti-high mobility group box 1 monoclonal antibody ameliorates brain infarction induced by transient ischemia in rats. FASEB J 2007;21:39043916.

24 Yang H, Hreggvidsdottir HS, Palmblad K, Wang H, Ochani M, Li J, Lu B, Chavan S, Rosas-Ballina M, Al-Abed Y, Akira S, Bierhaus A, Erlandsson-Harris H, Andersson U, Tracey KJ: A critical cysteine is required for HMGB1 binding to Toll-like receptor 4 and activation of macrophage cytokine release. Proc Natl Acad Sci USA 2010;107:1194211947.

25 Rosset A, Spadola L, Ratib O: OsiriX: An open-source software for navigating in multidimensional DICOM images. J Digit Imaging 2004;17:205-216.

26 Fournier T, Medjoubi NN, Porquet D: Alpha1-acid glycoprotein. Biochim Biophys Acta 2000;1482:157-171.

27 Tang Y, Zhao X, Antoine D, Xiao X, Wang H, Andersson U, Billiar TR, Tracey KJ, Lu B: Regulation of posttranslational modifications of HMGB1 during immune responses. Antioxid Redox Signal 2016;24:620-634.

28 Sasaki T, Liu K, Agari T, Yasuhara T, Morimoto J, Okazaki M, Takeuchi H, Toyoshima A, Sasada S, Shinko A, Kondo A, Kameda M, Miyazaki I, Asanuma M, Borlongan CV, Nishibori M, Date I: Anti-high mobility group box 1 antibody exerts neuroprotection in a rat model of Parkinson's disease. Exp Neurol 2016;275:220-231.

29 Norden DM, Muccigrosso MM, Godbout JP: Microglial priming and enhanced reactivity to secondary insult in aging, and traumatic CNS injury, and neurodegenerative disease. Neuropharmacology 2015;96:29-41.
30 Caso JR, Pradillo JM, Hurtado O, Lorenzo P, Moro MA, Lizasoain I: Toll-like receptor 4 is involved in brain damage and inflammation after experimental stroke. Circulation 2007; 115:1599-1608.

31 Yang QW, Lu FL, Zhou Y, Wang L, Zhong Q, Lin S, Xiang J, Li JC, Fang CQ, Wang JZ: HMBG1 mediates ischemia-reperfusion injury by TRIF-adaptor independent Toll-like receptor 4 signaling. J Cereb Blood Flow Metab 2011;31:593-605.

32 Lian YJ, Gong H, Wu TY, Su WJ, Zhang Y, Yang YY, Peng W, Zhang T, Zhou JR, Jiang CL, Wang YX: Ds-HMGB1 and fr-HMGB induce depressive behavior through neuroinflammation in contrast to nonoxid-HMGB1. Brain Behav Immun 2017;59:322-332.

-33 Frank MG, Weber MD, Fonken LK, Hershman SA, Watkins LR, Maier SF: The redox state of the alarmin HMGB1 is a pivotal factor in neuroinflammatory and microglial priming: a role for the NLRP3 inflammasome. Brain Behav Immun 2016;55:215-224.

34 Holmin S, Mathiesen T: Intracerebral administration of interleukin- $1 \beta$ and induction of inflammation, apoptosis, and vasogenic edema. J Neurosurg 2000;92:108-120.

35 Boutin H, LeFeuvre RA, Horai R, Asano M, Iwakura Y, Rothwell NJ: Role of IL- $1 \alpha$ and IL$1 \beta$ in ischemic brain damage. J Neurosci 2001; 21:5528-5534.

36 Garcia JH, Liu KF, Relton JK: Interleukin-1 receptor antagonist decreases the number of necrotic neurons in rats with middle cerebral artery occlusion. Am J Pathol 1995; 147:14771486.

37 Zandarashvili L, Sahu D, Lee K, Lee YS, Singh P, Rajarathnam K, Iwahara J: Real-time kinetics of high-mobility group box 1 (HMGB1) oxidation in extracellular fluids studied by in situ protein NMR spectroscopy. J Biol Chem 2013;288:11621-11627.

38 Venereau E, Schiraldi M, Uguccioni M, Bianchi ME: HMGB1 and leukocyte migration during trauma and sterile inflammation. Mol Immunol 2013;55:76-82.

39 Terrando N, Yang T, Wang X, Fang J, Cao M, Andersson U, Erlandsson HH, Ouyang W, Tong J: Systemic HMGB1 neutralization prevents postoperative neurocognitive dysfunction in aged rats. Front Immunol 2016;7:441.

40 Qin L, Wu X, Block ML, Liu Y, Breese GR, Hong JS, Knapp DJ, Crews FT: Systemic LPS causes chronic neuroinflammation and progressive neurodegeneration. Glia 2007;55: 453-462.
Extracellular HMGB1 Initiates CNS Inflammation 